\title{
Cortical Excitability in Chronic Migraine
}

\author{
Gianluca Coppola • Jean Schoenen
}

Published online: 12 November 2011

(C) Springer Science+Business Media, LLC 2011

\begin{abstract}
A proportion of episodic migraine patients experiences a progressive increase in attack frequency leading to chronic migraine (CM). The most frequent external factor that leads to headache chronification is medication overuse. The neurobiological bases of headache chronification and of the vicious circle of medication overconsumption are not completely elucidated. More recently, the same neurophysiological methods used to study episodic migraine were applied to $\mathrm{CM}$ and medication-overuse headache (MOH). Studies of cortical responsivity tend overall to indicate an increase in excitability, in particular of somatosensory and visual cortices, reflected by increased amplitude of evoked responses, decreased activity of inhibitory cortical interneurons reflected in the smaller magnetic suppression of perceptual accuracy, and, at least for visual responses, an increase in habituation. In $\mathrm{MOH}$, overconsumption of triptans or NSAIDs influences cortical excitability differently. Generalized central sensitization is suggested to play an important role in the pathophysiology of headache chronification.
\end{abstract}

Keywords Chronic migraine $\cdot$ Medication-overuse headache · Habituation - Sensitization - Cortical excitability . Evoked potentials . Transcranial magnetic stimulation . Chronic daily headache

\footnotetext{
G. Coppola

Department of Neurophysiology of Vision and

Neuroophthalmology, G.B. Bietti Eye Foundation-IRCCS, Rome, Italy

J. Schoenen $(\bowtie)$

Headache Research Unit, University Department

of Neurology \& GIGA Neurosciences, University of Liège,

Boulevard du 12ème de Ligne 1,

B-4000 Liège, Belgium

e-mail: jschoenen@ulg.ac.be
}

\section{Introduction}

Episodic migraine is characterized by the cyclic recurrence of headaches, separated by attack-free periods, of which the exact pathogenesis is still not completely known. Important insights into the pathophysiology of migraine were gained during the past decade, confirming that inherited and environmental factors are interrelated.

The genetic vulnerability to migraine, the so-called genetic migraine threshold, could be reflected phenotypically by increased responsiveness of the cerebral cortex to sensory information, dysfunctioning brainstem neurons, reduced mitochondrial energy reserve, and NO hypersensitivity among others.

A proportion of episodic migraine patients experiences a progressive increase in attack frequency leading to chronic migraine $(\mathrm{CM})$, defined as 15 or more headache days with 8 or more migraine attacks per month [1]. Various intrinsic and external factors can favor migraine chronification. The most frequent finding in clinical practice is medication-overuse headache $(\mathrm{MOH})$ that is diagnosed in up to $80 \%$ of patients attending specialized headache clinics [1-3]. Although any primary headache can evolve to $\mathrm{MOH}$, migraine is by far the most prevalent type. A history of frequent migraine and analgesic overuse are the most prominent risk factors for developing chronic daily headache $(\mathrm{CDH})[4,5]$. All known treatments taken for symptomatic headache relief, such as triptans, analgesics, and opioids, can induce transformation from episodic headache to $\mathrm{MOH}$, though NSAIDs seem less prone to do so $[1,3]$.

The neurobiological bases of headache chronification and of the vicious circle of medication overconsumption are not completely understood.

During the past decades, electrophysiological methods were particularly useful for the understanding of certain 
facets of migraine pathophysiology because they are able to detect functional brain abnormalities and they can be repeated noninvasively and at low cost. Most evoked-potential studies have shown that, for a number of different sensory modalities, the migrainous brain is characterized between attacks by deficient habituation brain potentials during stimulus repetition. This abnormality of cortical responsivity fluctuates over time in relation to the migraine attack; it is most pronounced during the days immediately preceding the attack and normalizes during the attack [6]. More recently, the same methods were applied to CM patients, and in particular to those with associated medication overuse.

This article focuses on the available neurophysiological data providing information about cortical responsiveness reactivity in patients who evolved from episodic migraine to $\mathrm{CM}$ with or without medication overuse.

\section{Published Data}

Although the precise mechanisms underlying headache chronification are not fully understood, central sensitization and dysfunctioning pain control systems are thought to play pivotal roles. The available published data are shown in Table 1.

\section{Pain-Related Responses}

Abnormalities of pain-related brain responses can be recorded in episodic migraine. It was assumed that these abnormalities would be even more marked in CM.

Brief radiant $\mathrm{CO}_{2}$ laser stimulations are able to activate small $\mathrm{A} \delta$ and $\mathrm{C}$ thermal nociceptors in superficial skin [7]. They have been used in the cranial or extracranial territories to elicit nociceptive evoked brain responses.

In episodic migraine, during the interictal phase, the negative-positive biphasic wave (N2-P2) laser-evoked potential (LEP) complex, which is chiefly generated in the anterior cingulate cortex, is normal in basal conditions after both cranial and extracranial stimulations. However, LEPs are abnormally modulated by heterotopic conditioning stimuli such as remote capsaicin application [8] and by distraction $[9,10]$.

By contrast, in a group of $25 \mathrm{CM}$ patients, a trend for an excessive increase in LEP N2-P2 amplitudes was detected at baseline [9]. This is less pronounced when the stimulus becomes painful and with increasing disease duration. The authors interpreted their results as evidence for a functional reorganization of the cortical pain matrix due to the attack repetition, a phenomenon similar to that described in other long-lasting pain conditions [9]. In fact, in a brain-mapping analysis of the cortical source of the most prominent LEP peak (P2), the same authors found that after supraorbital laser stimulation, $\mathrm{CM}$ patients were characterized by more pronounced activation of the rostral portion of the anterior cingulate cortex than episodic migraineurs and normal controls [11]. In addition, they observed that this positional shift of the P2 dipole, as expressed by its X-coordinate, was significantly correlated with headache frequency, but not with other clinical features or with greater self-evaluated depression and anxiety [11].

In a group of 29 migraine patients who evolved to $\mathrm{CDH}$ due to medication overuse, Ayzenberg et al. [12] recorded simultaneously blink reflex and pain-related evoked potentials (PREPs) after electrical stimulations of the forehead or the hand dorsum, respectively, with a planar concentric stimulating electrode selectively activating $\mathrm{A} \delta$ fibers. They observed a significant increase in PREP amplitudes in all patients with $\mathrm{MOH}$ whether they overused analgesics or triptans. PREP amplitudes were increased both after cephalic and extracephalic stimulation and were not related to self-perceived depressive mood. Discontinuation of the acute medication overuse tended to normalize the PREP amplitude [12]. By contrast, nociception-specific blink reflexes were normal, suggesting that sensitization takes place at the suprasegmental level in $\mathrm{MOH}$ patients.

\section{Non-noxious Somatosensory Evoked Potentials}

Somatosensory evoked potentials (SSEPs) are generated in afferent pathways, subcortical structures, and various regions of the cerebral cortex after galvanic stimulation of a peripheral nerve. SSEPs are obtained by weak sensory stimuli ideal for disclosing sensitization (reflected by an increased response amplitude to single or low numbers of stimuli) and habituation (reflected by a decrease in average response amplitude after high numbers of stimuli) [13, 14]. Using SSEPs, lack of both habituation and sensitization can be disclosed in migraine patients between attacks [15]. We recently studied SSEP sensitization and habituation in migraine patients with $\mathrm{MOH}$ $(n=29)$ by comparison with episodic migraine, recorded during $(n=23)$ and outside $(n=41)$ of an attack, and healthy subjects $(n=42$; Fig. 1a) [16••].

We managed to record SSEPs in $\mathrm{MOH}$ patients in a pain-free state or when experiencing only mild headaches. We observed that amplitude of the most prominent SSEP component, N20-P25, was initially (1st block) greater in $\mathrm{MOH}$ patients and in episodic migraineurs without aura studied ictally than in the subgroup studied interictally and healthy controls $\left[16 \bullet^{\bullet}\right]$. In control subjects and migraineurs without aura studied ictally, N20-P25 habituation was normal while it was clearly reduced in $\mathrm{MOH}$ and in migraine without aura patients recorded interictally. The increased SSEP amplitude in MOH was proportional to the duration of headache chronification. We interpreted these results as reflecting reinforcement and perpetuation of 
Table 1 Synopsis of published electrophysiological studies in chronic migraine and medication-overuse headache

\begin{tabular}{|c|c|c|c|c|}
\hline Study & Patients (with diagnosis), $n$ & $\begin{array}{l}\text { Neurophysiological } \\
\text { test }\end{array}$ & $\begin{array}{l}\text { Components } \\
\text { measured }\end{array}$ & Principal finding \\
\hline de Tommaso et al. [9] & $25 \mathrm{CM}$ & LEP & N2-P2 amplitude & $\begin{array}{l}\text { Nonsignificant trend for excessive increase in } \\
\text { N2-P2 amplitudes, less obvious with increasing } \\
\text { painfulness of the stimulus and longer disease } \\
\text { duration }\end{array}$ \\
\hline de Tommaso et al. [11] & $16 \mathrm{CM}$ & LEP & $\begin{array}{l}\text { Brain mapping analysis of } \\
\text { the cortical source of the } \\
\text { P2 peak }\end{array}$ & $\begin{array}{l}\text { Greater activation of the rostral portion of the } \\
\text { anterior cingulate cortex in CM patients } \\
\text { compared to EM and normal controls }\end{array}$ \\
\hline Ayzenberg et al. [12] & $\begin{array}{l}29 \mathrm{MOH}(14 \mathrm{NSAIDs} \\
\text { and } 14 \text { triptans) }\end{array}$ & PREP, nBR & N2-P2 amplitude & $\begin{array}{l}\text { Increase in PREP amplitudes in all patients with } \\
\mathrm{CM} \text {, normalizing after drug withdrawal. Normal } \\
\mathrm{nBR}\end{array}$ \\
\hline Coppola et al. $[16 \bullet \bullet]$ & $\begin{array}{l}29 \mathrm{MOH}(9 \text { triptans, } \\
10 \text { NSAIDs, and } 10 \text { both } \\
\text { medications combined) }\end{array}$ & SSEP & N20-P25 amplitude & $\begin{array}{l}\text { 1st block amplitudes greater in } \mathrm{MOH} \text { than in } \\
\text { interictal episodic migraine and healthy } \\
\text { volunteers. Habituation deficit in } \mathrm{MOH} \text { and } \\
\text { interictal migraine. Amplitudes smaller in triptan } \\
\text { overusers than in patients overusing NSAIDs or } \\
\text { both medications combined }\end{array}$ \\
\hline Siniatchkin et al. [18] & $15 \mathrm{TM}$ & $\mathrm{CNV}$ & $\begin{array}{l}\text { Amplitude of early } \\
\text { (CNV1) and late } \\
\text { (CNV2) components }\end{array}$ & $\begin{array}{l}\text { Low-amplitude CNV1 and lack of habituation In } \\
\text { TM. Reduction of CNV2 and increase of post- } \\
\text { imperative negative variation, the latter related to } \\
\text { depressive mood }\end{array}$ \\
\hline Chen et al. $[19 \bullet \bullet]$ & $25 \mathrm{CM}$ & VEF & P100m amplitude & $\begin{array}{l}\text { 1st block amplitude higher in CM and ictal EM } \\
\text { compared to interictal EM. Normal habituation } \\
\text { in CM, ictal EM, and control subjects, contrasting } \\
\text { with lack of habituation in interictal EM }\end{array}$ \\
\hline Chen et al. [20] & $18 \mathrm{CM}$ & VEF & P100m amplitude & $\begin{array}{l}\text { Normal habituation in CM patients. Lack of } \\
\text { habituation in patients with persistent visual aura }\end{array}$ \\
\hline Aurora et al. [22] & $25 \mathrm{CM}$ & TMS & MSPA & $\begin{array}{l}\text { Lower subjective phosphene threshold and smallest } \\
\text { visual suppression index in CM compared to EM } \\
\text { and control subjects }\end{array}$ \\
\hline Currà et al. [24] & $\begin{array}{l}40 \mathrm{MOH}(10 \text { triptans, } \\
13 \text { NSAIDs, and } \\
17 \text { combined })\end{array}$ & TMS & CSP duration & $\begin{array}{l}\text { CSP duration normal in MOH overall, while it was } \\
\text { reduced interictally in EM. CSP reduced in triptan } \\
\text { overusers compared to patients overusing } \\
\text { NSAIDs or triptans and NSAIDs combined. After } \\
\text { withdrawal, increase of CSP in combined } \\
\text { overusers; no change in triptan overusers }\end{array}$ \\
\hline
\end{tabular}

$C M$ chronic migraine, $C N V$ contingent negative variation, $C S P$ cortical silent period, $E M$ episodic migraine, $L E P$ laser evoked potentials, $N 2-P 2$ negative-positive biphasic wave, $n B R$ nociceptive specific blink reflex, NSAIDs nonsteroidal anti-inflammatory drugs, $M O H$ medication-overuse headache, MSPA magnetic suppression of perceptual accuracy, PREP pain-related evoked potentials, SSEP somatosensory evoked potentials, TM transformed migraine, TMS transcranial magnetic stimulation, $V E F$ visual evoked magnetic fields

central sensitization due to the medication overuse and increased headache frequency $[16 \bullet \cdot]$. As a further thoughtprovoking result, we noted that the SSEP changes differed according to the class of drugs overused; amplitudes were smaller in triptan overusers than in patients overusing NSAIDs or both medications combined, although both groups had the same lack of habituation during repeated stimulations.

\section{Contingent Negative Variation}

Contingent negative variation $(\mathrm{CNV})$ is a slow cortical potential related to higher mental functions, which consists of a negative wave generated in a reaction-time paradigm. Its early component (related to the warning stimulus) may represent the level of expectation and it is supposed to be modulated by the noradrenergic system, while its late component is thought to be related to motor readiness and to be under dopaminergic control [17].

Siniatchkin et al. [18] studied CNV amplitude and habituation in a group of 15 patients with $\mathrm{CDH}$ and analgesic overuse evolving from episodic migraine without aura (also called "transformed migraine") and for comparison in 15 episodic migraineurs and 15 healthy controls. Amplitude of total $\mathrm{CNV}$ and its early component was greater in episodic migraine patients than in $\mathrm{CDH}$ patients or controls. $\mathrm{CDH}$ patients had a smaller late $\mathrm{CNV}$ component and an enhanced post-imperative negative variation (PINV). The early $\mathrm{CNV}$ component did not habituate in episodic migraine or in $\mathrm{CDH}$ patients, but in the latter, this was related to significantly lower amplitudes. A positive relationship between PINV and self-rated depressive mood was found only in $\mathrm{CDH}$ patients [18]. According to the authors, these results reflect poor abilities 
A

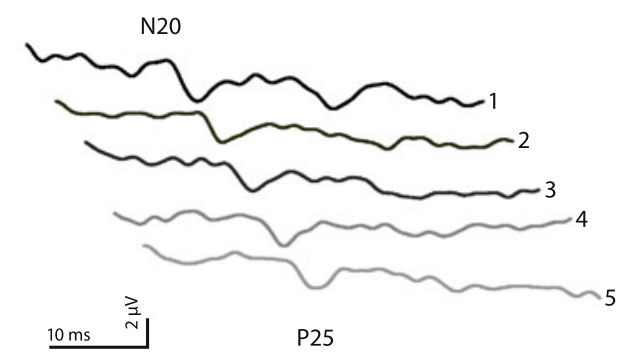

B

Healthy volunteer
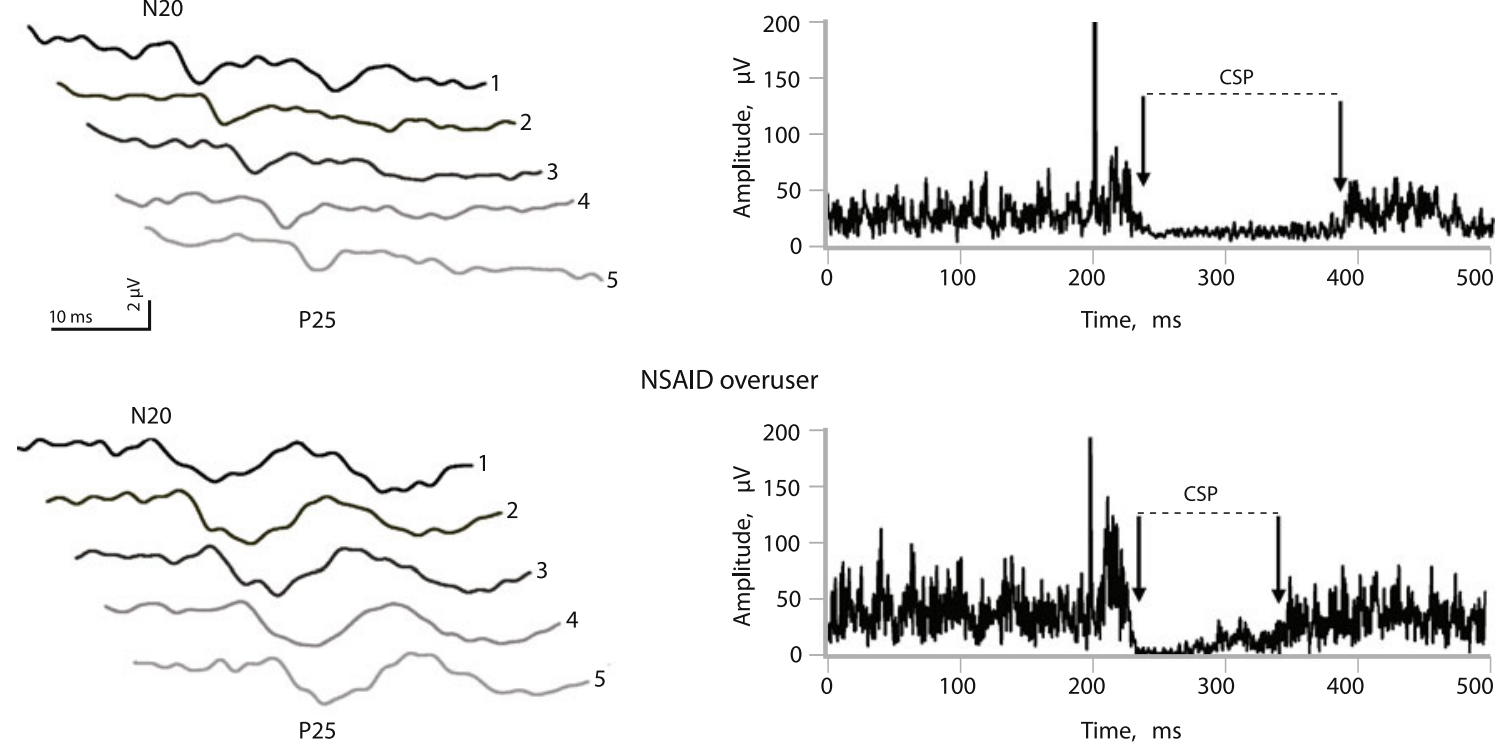

NSAID overuser

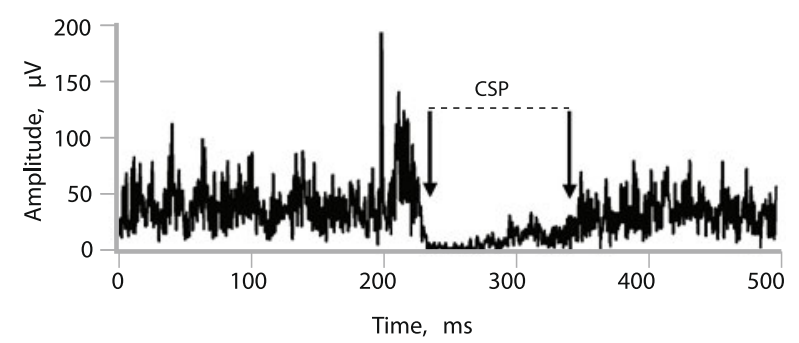

Triptan overuser
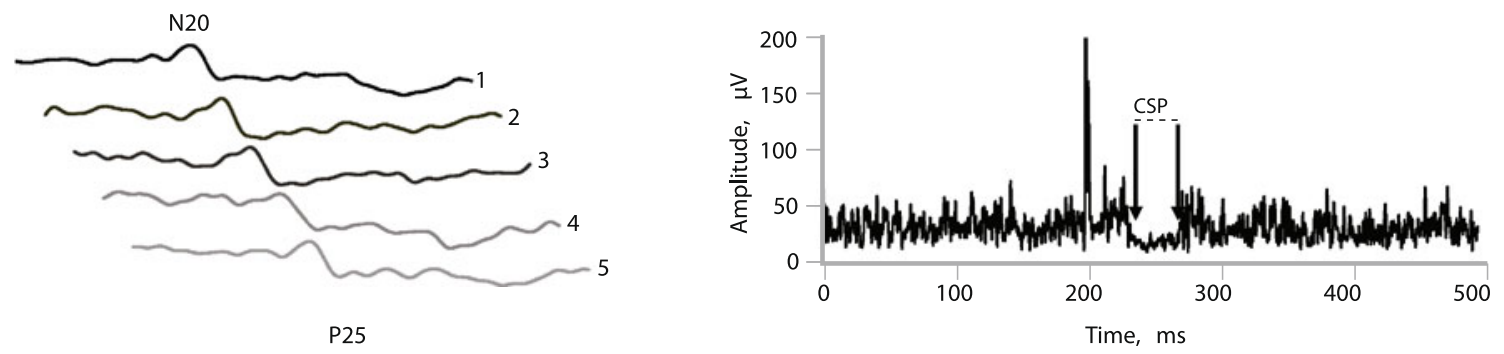

Fig. 1 Illustrative traces of a somatosensory evoked potentials habituation (SSEP) and b cortical silent period (CSP) in a healthy volunteer, an $\mathrm{MOH}$ patient overusing NSAIDs, and an $\mathrm{MOH}$ patient overusing a triptan. Note absence of SSEP habituation in both

patients, but increased amplitude in block 1 in the NSAID overuser contrasting with decreased amplitude in the triptan overuser. CSP is reduced in the former, but more so in the latter. $\mathrm{MOH}-$ medicationoveruse headache

to cope with environmental demands and susceptibility for depression in $\mathrm{CDH}$ patients. They also suggested that the late CNV component and PINV may be predictive variables for transformation of migraine.

\section{Visual Evoked Potentials}

The standard method for assessing excitability in the occipital cortex is to record visual evoked potentials (VEPs; ie, the electrical cortical response to structured visual stimuli). The occipital cortex lies outside the "pain matrix"; hence, it is supposedly unrelated to pain processing.

To search if the VEP abnormalities known to be present in episodic migraine interictally, notably lack of habituation, would be present in CM patients, Chen et al. [19••] recorded visual evoked magnetic fields (VEF) using magnetoencephalography (MEG) in $25 \mathrm{CM}$ patients and compared them to 38 episodic migraineurs (29 interictally, 9 ictally) and 32 healthy controls. CM patients were

recorded in a pain-free interval. Grand average $\mathrm{P} 100 \mathrm{~m}$ amplitudes as well as the 1 st block amplitude were significantly larger in CM patients and ictal episodic migraine patients compared to interictal episodic migraine subjects. On stimulus repetition, P100m amplitude habituated equally in CM patients, ictal episodic migraine patients, and control subjects, whereas a habituation deficit was recorded interictally in episodic migraineurs. In patients with $\mathrm{CM}$ and interictal episodic migraine, the $\mathrm{P} 100 \mathrm{~m}$ parameters did not correlate with any clinical variable [19••]. That VEF habituation is normal in CM $(n=18)$ was confirmed by the same authors in another study, where they found on the contrary that the VEF potentiates in patients with persistent visual aura $(n=6)[20]$.

\section{Transcranial Magnetic Stimulation}

Transcranial magnetic stimulation (TMS) allows noninvasive exploration of the excitability of certain cortical areas 
and durable modification via repetitive stimulations (rTMS).

In the method called magnetic suppression of perceptual accuracy (MSPA), a series of three-letter trigrams are presented on a computer screen and followed at variable intervals by a single TMS pulse over the occipital area that alters the perception accuracy. Aurora et al. [21], backing up their preliminary study of a small group of five patients with probable CM, applied the MSPA paradigm to study cortical inhibition in $25 \mathrm{CM}$ patients (with or without medication overuse) and, for comparison, in episodic migraine patients and healthy controls. CM patients had the lowest subjective phosphene threshold and the smallest suppression index, while healthy controls had the highest threshold and the largest suppression and episodic migraineurs fell in between those two groups [22]. Out of these $25 \mathrm{CM}$ patients, 10 underwent an $18 \mathrm{~F}$ fludeoxyglucose positron emission tomography scan, which showed increased metabolism in the pons and right temporal cortex, contrasting with decreased metabolism in bilateral medial frontal, parietal, and somatosensory areas and caudate nuclei [22].

Interestingly, considering that the dorsolateral prefrontal cortex (DLPFC) exerts an inhibitory control on pain pathways in humans, Brighina et al. [23] explored the effect of 12 daily sessions of high-frequency rTMS over the left DLPFC in a small sham-controlled study of $11 \mathrm{CM}$ patients. They found a long-lasting reduction in attack frequency, number of tablet intake, and headache index in the effectively treated $(n=6)$ compared to the sham-treated group $(n=5)$.

Assuming that medication overuse in headache patients alters the balance between inhibition and excitation in the cerebral cortex, we explored cortical inhibitory circuits by measuring the TMS-induced cortical silent period (CSP) [24]. The CSP is an interruption of voluntary muscle activity induced by a high-intensity magnetic pulse delivered over the motor cortex. The CSP elicited in facial muscles originates solely in the cortex [25]. CSP was recorded in $40 \mathrm{MOH}$ patients (10 triptan overusers, 13 NSAIDs overusers, and 17 triptan and NSAID overusers) and, for comparison, in 12 episodic migraineurs between attacks and 13 healthy subjects. CSP duration was reduced interictally in episodic migraineurs, but normal in the total group of $\mathrm{MOH}$ patients. However, analysis of subgroups showed that CSP duration was significantly shorter in triptan overusers than in the NSAID or triptan plus NSAID overuser groups (Fig. 1b). In 10 patients who overconsumed both triptans and NSAIDs, the CSP shortened and reached normal values 3 weeks after drug withdrawal. By contrast, in eight triptan overusers, there was no change in CSP duration after withdrawal. In $\mathrm{MOH}$ patients overall, CSP duration correlated positively with monthly tablet intake. However, this positive correlation was restricted to NSAID and triptan plus NSAID MOH subgroups; triptan overusers showed an inverse relationship [24].

\section{Conclusions}

To summarize, clinical neurophysiology has shown that cortical responsivity in migraine fluctuates over time not only in relation to the cycle of the migraine attacks (preictal/ictal/interictal), but also in relation to attack frequency. The most reproducible abnormality of evoked potentials, namely lack of habituation, is detectable during the pain-free interval and it is usually accompanied by a low response amplitude after low numbers of stimuli (1st block amplitude). By contrast, during the attack (ie, ictally), habituation and 1st block amplitude increase and normalize, while during the days immediately preceding the attack (ie, preictally), the habituation deficit further worsens. We have postulated that these cortical electrophysiological patterns are related to changes in serotonin transmission, which is low interictally [26], and may further decrease preictally but increase ictally, as suggested by several biochemical studies $[27,28]$ and numerous imaging studies $[29,30]$ showing activation of pontomesencephalic areas comprising monoaminergic nuclei during migraine attacks (see [6] for a review).

In $\mathrm{CM}$ patients, brainstem activation was found in similar locations as during migraine attacks $[5,22,31]$. Moreover, in $\mathrm{CM}$ with or without medication-overuse, electrophysiological studies have shown abnormalities that are also reported during attacks in episodic migraineurs (Table 2). These abnormalities tend overall to indicate an increase in cortical excitability, in particular of somatosensory and visual cortices, reflected by an increased amplitude of evoked cortical responses, decreased activity of inhibitory cortical interneurons reflected in the smaller MSPA [22], and, at least for VEPs, an increase in habituation. This suggests that from an electrophysiological point of view, CM looks like a "never ending migraine attack" [32].

We have shown before that 1st block amplitude and habituation are directly correlated as far as VEPs are concerned [33]. This appears to hold true in CM without drug overuse for visual evoked responses, but not in $\mathrm{MOH}$ for SSEP [16••], where amplitude was increased and habituation decreased. The discrepancy between the two studies could be due to the fact that they explore two different sensory systems and that plastic changes occurring during chronic headache predominate in areas belonging to the pain matrix, and thus, in the somatosensory cortex. However, a more likely explanation is that the difference is due to brain effects of the prolonged overuse of drugs in the 
Table 2 Synoptic table of electrophysiological changes comparing episodic migraine (between and during attacks) and chronic migraine with or without medication overuse

\begin{tabular}{|c|c|c|c|c|c|c|}
\hline & \multicolumn{2}{|c|}{ Episodic migraine (interictal) } & \multicolumn{2}{|c|}{ Episodic migraine (ictal) } & \multicolumn{2}{|c|}{ Chronic migraine/MOH } \\
\hline & Amplitude & Habituation & Amplitude & Habituation & Amplitude & Habituation \\
\hline \multicolumn{7}{|l|}{ LEP } \\
\hline Trigeminal & $\mathrm{N}$ & $\downarrow$ & $\uparrow$ & $\uparrow$ & $\uparrow$ & \\
\hline \multicolumn{7}{|l|}{ PREP } \\
\hline Trigeminal & & & & & $\uparrow$ & \\
\hline Extracephalic & & & & & $\uparrow$ & \\
\hline NSBR & $\mathrm{N}$ & $\downarrow$ & $\uparrow$ & $\uparrow$ & $\mathrm{N}$ & \\
\hline \multirow[t]{2}{*}{ SSEP } & $\downarrow$ (1st block) & $\downarrow$ & $\uparrow$ & $\uparrow$ & $\uparrow$ (NSAIDs) & $\downarrow$ \\
\hline & & & & & $\mathrm{N}$ (triptans) & \\
\hline \multicolumn{7}{|l|}{$\mathrm{CNV}$} \\
\hline Early & $\uparrow$ & $\downarrow$ & $\downarrow$ & $\uparrow$ & $\downarrow$ (1st block $)$ & $\downarrow(\mathrm{PINV} \uparrow)$ \\
\hline Late & $\mathrm{N}$ & $\mathrm{N}$ & & & $\downarrow$ & (BDI: 21) \\
\hline \multicolumn{7}{|l|}{ VEP } \\
\hline Electric & $\downarrow$ (1st block) & $\downarrow$ & $\uparrow(1$ st block) & $\uparrow$ & $\uparrow$ & $\uparrow$ \\
\hline Magnetic & $\downarrow$ & $\downarrow$ & $\uparrow$ & $\uparrow$ & & \\
\hline \multirow[t]{2}{*}{ Cortical silent period (TMS) } & $\downarrow$ & & & & N (NSAIDs) & \\
\hline & & & & & $\downarrow$ (triptans) & \\
\hline
\end{tabular}

The differences between chronic migraine patients with and without medication overuse described in the text are not shown

Arrows indicate the direction of change, $B D I$ Beck Depression Inventory score, $C N V$ contingent negative variation, $L E P$ laser evoked potential, $M O H$ medication-overuse headache, $N$ normal, $N S A I D$ s nonsteroidal anti-inflammatory drugs, $N S B R$ nociception-specific blink reflex, $P I N V$ postimperative negative variation, $P R E P$ pain-related evoked potential, SSEP somatosensory evoked potential, TMS transcranial magnetic stimulation, $V E P$ visual evoked potential

SSEP study. This also may explain why habituation remains abnormal in the $\mathrm{CNV}$ study of patients with $\mathrm{MOH}$ [18]. Our observation that the increased grand average SSEP amplitude in $\mathrm{MOH}$ was proportional to the duration of the chronic headache phase [16••] would favor this explanation, as does our unpublished finding that SSEP habituation is normal in CM patients without medication overuse.

From a physiological point of view, evoked-potential amplitude to low numbers of stimuli is a marker of sensitization, while amplitude reduction over large numbers of stimuli reflects habituation (or adaptation). Both phenomena are considered to be partially independent and, therefore, can be differentially affected by pharmacological interventions [14, 42]. As a matter of fact, in our $\mathrm{MOH}$ patients, NSAIDs overuse induced SSEP sensitization (increased 1st amplitude block) that persisted over time (lack of habituation), while triptans reduced both sensitization and habituation, although we suspect that in this case, the reduction in habituation is chiefly due to the low initial amplitudes causing a "low ceiling" effect [16••]. We also have found that triptans and NSAIDs, when overused, differently influence excitability of the somatosensory and the motor cortices. While NSAID overuse appears to promote sensitization in the sensory system, triptan overuse does not, but it reduces inhibitory mechanisms in the motor cortex in proportion to the degree of overconsumption [24]. The biological explanation for this difference is not known, but it is likely related to the different pharmacological actions and targets of these drugs. The clinical differences between NSAID- and triptan-overuse headaches are well known [43] and they were shown to have different effects on the trigeminal system after repeated administration in experimental animals [44]. It remains to be shown if there is a relation between experimental findings and clinical features. Meanwhile, it is of interest that NSAID overuse induces sensitization in the somatosensory system and that it also is associated with a longer duration of withdrawal headaches compared to triptans [45].

Increased amplitude of non-noxious (SSEP and VEP) and noxious (LEP and PREP) evoked responses probably reflect central sensitization. Central sensitization may indeed play an important role in the pathophysiology of headache chronification. While it is supposed to occur transiently during the migraine attack and to manifest itself clinically as cutaneous allodynia [34] and neurophysiologically as an increase in trigeminal reflexes [35], there is some evidence that it may be more persistent in $\mathrm{CM}$ where allodynia, for example, is highly prevalent [36, 37]. It has been hypothesized that a condition characterized by a continuous painful state, such as the daily headache of $\mathrm{CM}$ and $\mathrm{MOH}$ patients, leads to a permanent dysfunction of the 
antinociceptive modulatory pathways [38]. Central sensitization could be associated with plastic changes in various cerebral areas belonging to the so-called pain matrix [39, 40] that could be reflected neurophysiologically by the anatomical shifts found in the pain-activated cortical areas in the previously described LEP study of CM patients [11]. Central sensitization in the pain network is associated with abnormal neuronal excitability, causing a decrease in nociceptive thresholds, an increased responsiveness to both noxious and innocuous peripheral stimuli, and an expansion of the receptive fields of nociceptors [41]. It is of pathophysiological interest that central sensitization, as suggested by increased amplitudes of PREPs, seems to be generalized and suprasegmental because nociception-specific blink reflexes were normal in $\mathrm{MOH}$ patients $[9,12]$.

VEF habituation in CM [19••, 20] and SSEP lack of habituation in $\mathrm{MOH}[16 \cdot \bullet$ ] are independent of the presence or intensity of headache at the time of recording. This clearly argues against the hypothesis that cortical changes in $\mathrm{CM} / \mathrm{MOH}$ are a nonspecific consequence of ongoing pain [46].

To conclude, the methods of clinical neurophysiology are particularly appropriate to improve noninvasively our understanding of the cortical and brainstem dysfunctions associated with migraine and with its chronification by medication overuse. However, further research is necessary and should include more detailed studies taking into account the differences between pharmacological classes and between migraine chronification with and without medication overuse. New insight also can be gained by studies combining electrophysiology and functional brain imaging.

Disclosures No potential conflicts of interest relevant to this article were reported.

\section{References}

Papers of particular interest, published recently, have been highlighted as:

•- Of major importance

1. Olesen J, Bousser MG, Diener HC, et al. New appendix criteria open for a broader concept of chronic migraine. Cephalalgia. 2006;26(6):742-6.

2. Diener HC, Limmroth V. Medication-overuse headache: a worldwide problem. Lancet Neurol. 2004;3(8):475-83.

3. Silberstein SD, Olesen J, Bousser MG, et al. International Headache Society: the International Classification of Headache Disorders, 2nd Edition (ICHD-II)-revision of criteria for 8.2 Medication-overuse headache. Cephalalgia. 2005;25(6):460-5.

4. Wang SJ, Fuh JL, Lu SR, et al. Chronic daily headache in Chinese elderly: prevalence, risk factors, and biannual follow-up. Neurology. 2000;54(2):314-9.
5. Bigal ME, Lipton RB. Modifiable risk factors for migraine progression. Headache. 2006;46(9):1334-43.

6. Coppola G, Pierelli F, Schoenen J. Is the cerebral cortex hyperexcitable or hyperresponsive in migraine? Cephalalgia. 2007;27(12):1427-39.

7. Treede R, Lorenz J, Baumgärtner U. Clinical usefulness of laserevoked potentials. Neurophysiol Clin. 2003;33(6):303-14.

8. de Tommaso M, Difruscolo O, Sardaro M, et al. Effects of remote cutaneous pain on trigeminal laser-evoked potentials in migraine patients. J Headache Pain. 2007;8(3):167-74.

9. de Tommaso M, Valeriani M, Guido M, et al. Abnormal brain processing of cutaneous pain in patients with chronic migraine. Pain. 2003;101(1-2):25-32.

10. de Tommaso M, Baumgartner U, Sardaro M, et al. Effects of distraction versus spatial discrimination on laser-evoked potentials in migraine. Headache. 2008;48(3):408-16.

11. de Tommaso M, Losito L, Difruscolo O, et al. Changes in cortical processing of pain in chronic migraine. Headache. 2005;45(9):1208-18.

12. Ayzenberg I, Obermann M, Nyhuis P, et al. Central sensitization of the trigeminal and somatic nociceptive systems in medication overuse headache mainly involves cerebral supraspinal structures. Cephalalgia. 2006;26(9):1106-14.

13. Groves PM, Thompson RF. Habituation: a dual-process theory. Psychol Rev. 1970;77(5):419-50.

14. Rankin $\mathrm{CH}$, Abrams T, Barry RJ, et al. Habituation revisited: an updated and revised description of the behavioral characteristics of habituation. Neurobiol Learn Mem. 2009;92(2):135-8.

15. Ozkul Y, Uckardes A. Median nerve somatosensory evoked potentials in migraine. Eur J Neurol. 2002;9(3):227-32.

16. • Coppola G, Currà A, Di Lorenzo $\mathrm{C}$, et al: Abnormal cortical responses to somatosensory stimulation in medication-overuse headache. BMC Neurol 2010, 10:126. This study showed that the somatosensory cortex becomes persistently sensitized with medication overuse. The sensitization is obvious in patients overusing NSAIDs and almost nonexistent or masked in those who overuse only triptans.

17. Birbaumer N, Elbert T, Canavan AG, Rockstroh B. Slow potentials of the cerebral cortex and behavior. Physiol Rev. 1990;70(1):1-41.

18. Siniatchkin M, Gerber WD, Kropp P, Vein A: Contingent negative variation in patients with chronic daily headache. Cephalalgia 1998, 18(8):565-9; discussion 531.

19. •• Chen WT, Wang SJ, Fuh JL, et al: Persistent ictal-like visual cortical excitability in chronic migraine. Pain. 2011, 152(2):254-258. This study showed, for the first time, that the response pattern of the visual cortex in patients with CM is similar to that found during a common migraine attack in patients with episodic migraine.

20. Chen WT, Lin YY, Fuh JL, et al. Sustained visual cortex hyperexcitability in migraine with persistent visual aura. Brain. 2011;134(Pt 8):2387-95.

21. Aurora S, Barrodale P, Chronicle E, Mulleners W. Cortical inhibition is reduced in chronic and episodic migraine and demonstrates a spectrum of illness. Headache. 2005;45(5):546-52.

22. Aurora S, Barrodale P, Tipton R, Khodavirdi A. Brainstem dysfunction in chronic migraine as evidenced by neurophysiological and positron emission tomography studies. Headache. 2007;47 (7):996-1003.

23. Brighina F, Piazza A, Vitello G, et al. rTMS of the prefrontal cortex in the treatment of chronic migraine: a pilot study. J Neurol Sci. 2004;227(1):67-71.

24. Currà $\mathrm{A}$, Coppola $\mathrm{G}$, Gorini $\mathrm{M}$, et al. Drug-induced changes in cortical inhibition in medication overuse headache. Cephalalgia. 2011;31(12):1282-90.

25. Cruccu G, Inghilleri M, Berardelli A, et al. Cortical mechanisms mediating the inhibitory period after magnetic stimulation of the facial motor area. Muscle Nerve. 1997;20(4):418-24. 
26. Panconesi A. Serotonin and migraine: a reconsideration of the central theory. J Headache Pain. 2008;9(5):267-76.

27. Ferrari MD, Saxena PR. On serotonin and migraine: a clinical and pharmacological review. Cephalalgia. 1993;13(3):151-65.

28. Evers S, Quibeldey F, Grotemeyer KH, et al. Dynamic changes of cognitive habituation and serotonin metabolism during the migraine interval. Cephalalgia. 1999;19(5):485-91.

29. Weiller C, May A, Limmroth V, et al. Brain stem activation in spontaneous human migraine attacks. Nat Med. 1995;1(7):658 60 .

30. Bahra A, Matharu MS, Buchel C, et al. Brainstem activation specific to migraine headache. Lancet. 2001;357(9261):1016-7.

31. Matharu MS, Bartsch T, Ward N, et al. Central neuromodulation in chronic migraine patients with suboccipital stimulators: a PET study. Brain. 2004;127(Pt 1):220-30.

32. Schoenen J. Is chronic migraine a never-ending migraine attack? Pain. 2011;152(2):239-40.

33. Afra J, Proietti Cecchini A, Sándor PS, Schoenen J. Comparison of visual and auditory evoked cortical potentials in migraine patients between attacks. Clin Neurophysiol. 2000;111(6):1124-9.

34. Burstein R, Cutrer MF, Yarnitsky D. The development of cutaneous allodynia during a migraine attack clinical evidence for the sequential recruitment of spinal and supraspinal nociceptive neurons in migraine. Brain. 2000;123(Pt 8):1703-9.

35. Kaube H, Katsarava Z, Przywara S, et al. Acute migraine headache: possible sensitization of neurons in the spinal trigeminal nucleus? Neurology. 2002;58(8):1234-8.

36. Lovati C, D'Amico D, Bertora $\mathrm{P}$, et al. Acute and interictal allodynia in patients with different headache forms: an Italian pilot study. Headache. 2008;48(2):272-7.
37. Bigal ME, Ashina S, Burstein R, et al. Prevalence and characteristics of allodynia in headache sufferers: a population study. Neurology. 2008;70(17):1525-33.

38. Dodick D, Silberstein S. Central sensitization theory of migraine: clinical implications. Headache. 2006;4:S182-91.

39. Schmidt-Wilcke T, Leinisch E, Straube A, et al. Gray matter decrease in patients with chronic tension type headache. Neurology. 2005;65(9):1483-6.

40. Fumal A, Laureys S, Di Clemente L, et al. Orbitofrontal cortex involvement in chronic analgesic-overuse headache evolving from episodic migraine. Brain. 2006;129(Pt 2):543-50.

41. Woolf CJ, Wall PD. Relative effectiveness of C primary afferent fibers of different origins in evoking a prolonged facilitation of the flexor reflex in the rat. J Neurosci. 1986;6(5):1433-42.

42. Thompson RF, Spencer WA. Habituation: a model phenomenon for the study of neuronal substrates of behavior. Psychol Rev. 1966;73(1):16-43.

43. Limmroth V, Katsarava Z, Fritsche G, et al. Features of medication overuse headache following overuse of different acute headache drugs. Neurology. 2002;59(7):1011-4.

44. De Felice M, Ossipov MH, Wang R, et al. Triptan-induced enhancement of neuronal nitric oxide synthase in trigeminal ganglion dural afferents underlies increased responsiveness to potential migraine triggers. Brain. 2010;133(Pt 8):2475-88.

45. Katsarava Z, Fritsche G, Muessig M, et al. Clinical features of withdrawal headache following overuse of triptans and other headache drugs. Neurology. 2001;57(9):1694-8.

46. Stankewitz A, May A. The phenomenon of changes in cortical excitability in migraine is not migraine-specific-a unifying thesis. Pain. 2009;145(1-2):14-7. 